\title{
Discrete Versions of Stokes' Theorem Based on Families of Weights on Hypercubes
}

\author{
Gilbert Labelle and Annie Lacasse \\ Laboratoire de Combinatoire et d'Informatique Mathématique, UQAM \\ CP 8888, Succ. Centre-ville, Montréal (QC) Canada H3C3P8 \\ Laboratoire d'Informatique, de Robotique et de Microélectronique de Montpellier, \\ 161 rue Ada, 34392 Montpellier Cedex 5 - France \\ labelle.gilbert@uqam.ca, annie.lacasse@lirmm.fr
}

\begin{abstract}
This paper generalizes to higher dimensions some algorithms that we developed in [123] using a discrete version of Green's theorem. More precisely, we present discrete versions of Stokes' theorem and Poincaré lemma based on families of weights on hypercubes. Our approach is similar to that of Mansfield and Hydon [4] where they start with a concept of difference forms to develop their discrete version of Stokes' theorem. Various applications are also given.
\end{abstract}

Keywords: Discrete Stokes' theorem, Poincaré lemma, hypercube.

\section{Introduction}

Typically, physical phenomenon are described by differential equations involving differential operators such as divergence, curl and gradient. Numerical approximation of these equations is a rich subject. Indeed, the use of simplicial or (hyper)cubical complexes is well-established in numerical analysis and in computer imagery. Such complexes often provide good approximations to geometrical objects occuring in diverse fields of applications (see Desbrun and al. [5] for various examples illustrating this point). In particular, discretizations of differential forms occuring in the classical Green's and Stokes' theorems allows one to reduce the (approximate) computation of parameters associated with a geometrical object to computation associated with the boundary of the object (see 4 $45\left[67^{\prime}\right)$.

In the present paper, we focus our attention on hypercubic configurations and systematically replace differential forms by weight functions on hypercubes. We extend the classical difference and summation operators $(\Delta, \Sigma)$, of the finite difference calculus, to weight functions on hypercubic configurations in order to formulate our discrete versions of Stokes' theorem. For example, using these operators, the total volume, coordinates of the center of gravity, moment of inertia and other statistics of an hypercubic configuration can be computed using suitable weight functions on its boundary (see Section 4 below).

Although our approach have connections with the works [46] on cubical complexes and cohomology in $\mathbb{Z}^{n}$, we think that it can bridge a gap between differential calculus and discrete geometry since no differential forms or operations 
of standard exterior algebra calculus are involved. Moreover, our results are easily implemented on computer algebra systems such as Maple or Mathematica since families of weight functions on hypercubes, difference and summation operators are readily programmable. Finally, weight functions can take values in an arbitrary commutative ring. In particular, taking values in the boolean ring $B=\{0,1$, and, xor $\}$, our results provide algorithms to decide, for example, whether a given hypercube (or pixel, in the bi-dimensional case) belongs to an hypercubic configuration (or polyomino) using a suitable weight on its boundary (see $1 / 2] 3$, for similar examples in the case of polyominoes).

For completeness, in Section 2 we state the classical Stokes' theorem and recall some elementary concepts on differential forms. Section 3 describes discrete geometrical objects such as hypercubes, hypercubic configurations together with exterior difference and summation operators on weight functions which are used to formulate our discrete versions of Stokes' theorem. Section 4 illustrates the theory through examples. Due to the lack of space, most of the proofs (see [3]) are omitted in this extended abstract.

\section{Classical Differential Forms and Stokes' Theorem}

We start our analysis with the following version of Stokes' theorem [8]

Theorem 1. Let $\omega$ be a differential $(k-1)$-form and $K$ a $k$-surface in $\mathbb{R}^{n}$ with positively oriented boundary $\partial K$. Then,

$$
\int_{K} d \omega=\int_{\partial K} \omega
$$

where $d$ is the exterior differential operator.

In this statement, we suppose implicitly that $\omega$ is sufficiently differentiable and $K$ sufficiently smooth. Moreover, a differential $(k-1)$-form $\omega$ can be written in the standard form

$$
\omega=\sum_{1 \leq j_{1}<\cdots<j_{k-1} \leq n} \omega_{j_{1}, \ldots, j_{k-1}}\left(x_{1}, \ldots, x_{n}\right) d x_{j_{1}} \wedge \cdots \wedge d x_{j_{k-1}}
$$

and its exterior derivative, $d \omega$ is a $k$-form described by

$$
d \omega=\sum_{1 \leq j_{1}<\cdots<j_{k-1} \leq n}\left(d \omega_{j_{1}, \ldots, j_{k-1}}\left(x_{1}, \ldots, x_{n}\right)\right) \wedge d x_{j_{1}} \wedge \cdots \wedge d x_{j_{k-1}}
$$

where $d f\left(x_{1}, \ldots, x_{n}\right)=\frac{\partial f}{\partial x_{1}} d x_{1}+\cdots+\frac{\partial f}{\partial x_{n}} d x_{n}$. Making use of the general formulas $d x_{j} \wedge d x_{i}=-d x_{i} \wedge d x_{j}$, we obtain,

$$
d \omega=\sum_{1 \leq i_{1}<\cdots<i_{k} \leq n}(d \omega)_{i_{1}, \ldots, i_{k}}\left(x_{1}, \ldots, x_{n}\right) d x_{i_{1}} \wedge \cdots \wedge d x_{i_{k}},
$$


where component $(d \omega)_{i_{1}, \ldots, i_{k}}$ is the function of $\left(x_{1}, \ldots, x_{n}\right)$, given by

$$
(d \omega)_{i_{1}, \ldots, i_{k}}=\sum_{\nu=1}^{k}(-1)^{\nu-1} \frac{\partial \omega_{i_{1}, \ldots, \hat{i_{\nu}}, \ldots, i_{k}}}{\partial x_{i_{\nu}}},
$$

where $\widehat{i_{\nu}}$ means that index $i_{\nu}$ is omitted. One of the fundamental properties of the exterior differential operator is $d^{2}=0$, meaning that for every differential form $\omega$, we have $d(d \omega)=0$. We now recall some basic notions and facts about differential forms. A differential form $\omega$ satisfying $d \omega=0$ is said to be closed. Moreover, if there exists another form $\eta$, such that $\omega=d \eta$, then we say that $\omega$ is exact. The following result is immediate: If $\omega$ is exact then $\omega$ is closed. Poincaré lemma states that, under some conditions, the converse is also true. In this work, the following version of Poincaré lemma [8] will be sufficient:

Lemma 1. Let $\omega$ be a $k$-form with $k \geq 1$. If $\omega$ is closed and defined on a starshaped open set of $\mathbb{R}^{n}$, then $\omega$ is exact. Hence, $\omega=d \eta \Longleftrightarrow d \omega=0$.

In particular, if $\omega$ is defined everywhere on $\mathbb{R}^{n}$ then the notions of closedness and exactness coincide and no cohomology issues occur.

\section{Discretization of Stokes' Theorem}

A first step in our discretization is to notice that $K$ may have corners which can be seen as limiting cases of continuously differentiable round corners. This leads to consider the case where $K$ is a $k$-dimensional hypercube in $\mathbb{R}^{n}$. In this context, Mansfield and Hydon [4] have replaced differential forms

$$
\sum_{1 \leq i_{1}<\cdots<i_{k} \leq n} \omega_{i_{1}, \ldots, i_{k}}\left(x_{1}, \ldots, x_{n}\right) d x_{i_{1}} \wedge \cdots \wedge d x_{i_{k}},
$$

where $\left(x_{1}, \ldots, x_{n}\right) \in \mathbb{R}^{n}$, by difference forms

$$
\sum_{1 \leq i_{1}<\cdots<i_{k} \leq n} \omega_{i_{1}, \ldots, i_{k}}\left(x_{1}, \ldots, x_{n}\right) \Delta_{i_{1}} \wedge \cdots \wedge \Delta_{i_{k}},
$$

where $\left(x_{1}, \ldots, x_{n}\right) \in \mathbb{Z}^{n}$ instead of $\mathbb{R}^{n}$ and $\Delta_{i}=\Delta_{x_{i}}$ for $i=1, \ldots, n$ denote the finite partial difference operators with respect to the $i$-th component, that is $\Delta_{i} u\left(x_{1}, \ldots, x_{n}\right)=u\left(x_{1}, \ldots, x_{i}+1, \ldots, x_{n}\right)-u\left(x_{1}, \ldots, x_{i}, \ldots, x_{n}\right)$ for every function $u=u\left(x_{1}, \ldots, x_{n}\right)$.

We propose a variant of this approach in which the difference forms are systematically replaced by families of weights defined on hypercubes. First, consider the set

$\mathcal{H}_{k, n}=\left\{H \mid H\right.$ is a unit hypercube $\subseteq \mathbb{R}^{n}$ of $\operatorname{dim} k$ with vertices in $\left.\mathbb{Z}^{n}\right\}$. An $h y$ percube $H \in \mathcal{H}_{k, n}$ can be seen as a generalized pixel and is denoted

$$
\begin{aligned}
H & =\operatorname{Pix}_{i_{1}, \ldots, i_{k}}\left(\alpha_{1}, \ldots, \alpha_{n}\right) \\
& =\left\{\begin{array}{ll}
\left(x_{1}, \ldots, x_{n}\right) & \begin{array}{l}
x_{j}=\alpha_{j}, \\
\alpha_{j} \leq x_{j} \leq \alpha_{j}+1,
\end{array} \quad \text { if } j \notin\left\{i_{1}, \ldots, i_{k}\right\} \\
\left.\alpha_{1}, \ldots, i_{k}\right\}
\end{array}\right\},
\end{aligned}
$$


where $\left(\alpha_{1}, \ldots, \alpha_{n}\right) \in \mathbb{Z}^{n}$ and $\left\{i_{1}<\cdots<i_{k}\right\} \subseteq\{1, \ldots, n\}$ denotes the ordered set of indices of coordinates varying in the hypercube. We say that the hypercube $H$ comes from the point $\left(\alpha_{1}, \ldots, \alpha_{n}\right)$ according to the directions $i_{1}, \ldots, i_{k}$. The point $\left(\alpha_{1}, \ldots, \alpha_{n}\right)$ is also called principal corner of the hypercube and the two orientations of $H$ are denoted $H$ and $-H$. Using this convention, an hypercubic configuration denoted $\mathbf{P}$, of dimension $k$ in $\mathbb{R}^{n}$, with vertices in $\mathbb{Z}^{n}$, is defined as a finite $\mathbb{Z}$-linear combination of hypercubes $\in \mathcal{H}_{k, n}$ and $\mathbb{Z} \mathcal{H}_{k, n}$ denotes the set of hypercubic configurations of dimension $k$ in $\mathbb{R}^{n}$. Changing sign corresponds to changing orientation. Obviously, if all the coefficients are equal to 1, an hypercubic configuration is interpreted as a union of hypercubes. In the general case, an hypercubic configuration can be interpreted as a union of hypercubes with several multiplicities and orientations. Since the boundary of a region is a natural way to describe objects, we define the boundary operator $\partial$ as follows.

Definition 1. The boundary $\partial H$ of an hypercube $H=\operatorname{Pix}_{i_{1}, \ldots, i_{k}}\left(\alpha_{1}, \ldots, \alpha_{n}\right)$ is the hypercubic configuration

$$
\partial H=\partial \operatorname{Pix}_{i_{1}, \ldots, i_{k}}\left(\alpha_{1}, \ldots, \alpha_{n}\right)=\sum_{\nu=1}^{k}(-1)^{\nu-1} \Delta_{i_{\nu}} \operatorname{Pix}_{i_{1}, \ldots, \hat{i_{\nu}}, \ldots, i_{k}}\left(\alpha_{1}, \ldots, \alpha_{n}\right) .
$$

More generally, by linearity, the boundary of an hypercubic configuration $\mathbf{P}=$ $\sum_{j} n_{j} H_{j}, n_{j} \in \mathbb{Z}$ is defined by $\partial \mathbf{P}=\sum_{j} n_{j} \partial H_{j}$.

For example, let $n=k=2$ and $\mathbf{P}=\operatorname{Pix}_{1,2}(1,1)+\operatorname{Pix}_{1,2}(2,1)+\operatorname{Pix}_{1,2}(1,2)$, then, after simplifications,

$$
\begin{aligned}
\partial \mathbf{P}= & \operatorname{Pix}_{1}(1,1)+\operatorname{Pix}_{1}(2,1)+\operatorname{Pix}_{2}(3,1)-\operatorname{Pix}_{1}(2,2)+\operatorname{Pix}_{2}(2,2) \\
& -\operatorname{Pix}_{1}(1,3)-\operatorname{Pix}_{2}(1,2)-\operatorname{Pix}_{2}(1,1),
\end{aligned}
$$

which corresponds to Figure 1(a). This figure can be reinterpreted as a conventional oriented path as in Figure 1(b).

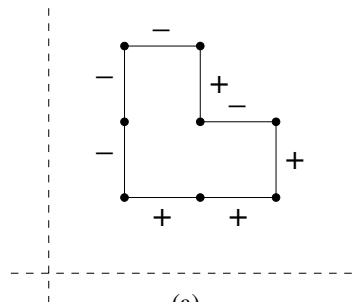

(a)

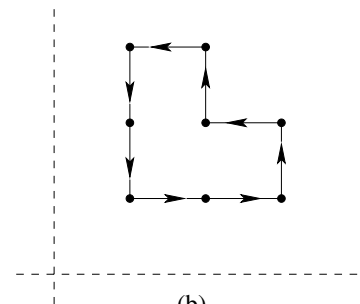

(b)

Fig. 1. Boundary: (a) linear combination of segments (b) oriented path

Since every differential $k$-form $\omega$ gives rise to a weight function $w$ defined on hypercubes of dimension $k$ by $w(H)=\int_{H} \omega$, this allows us to replace integrals by weight functions on hypercubes in the following way. 
Definition 2. An arbitrary $w: \mathcal{H}_{k, n} \longrightarrow \mathbb{R}$ is called a weight function on $k$ dimensional hypercubes in $\mathbb{R}^{n}$.

Note that such a weight function is equivalent to a family $\left(w_{j_{1}, \ldots, j_{k}}\right)_{1 \leq j_{1}<\cdots<j_{k} \leq n}$ made of $\left(\begin{array}{l}n \\ k\end{array}\right)$ functions $w_{j_{1}, \ldots, j_{k}}: \mathbb{Z}^{n} \longrightarrow \mathbb{R}$ defined by

$$
\left(\alpha_{1}, \ldots, \alpha_{n}\right) \mapsto w_{j_{1}, \ldots, j_{k}}\left(\alpha_{1}, \ldots, \alpha_{n}\right)=w\left(\operatorname{Pix}_{j_{1}, \ldots, j_{k}}\left(\alpha_{1}, \ldots, \alpha_{n}\right)\right) .
$$

More generally, we can take functions $w_{j_{1}, \ldots, j_{k}}: \mathbb{Z}^{n} \longrightarrow \mathbb{A}$, where $\mathbb{A}$ is an arbitrary commutative ring or a vector space. For example, $\mathbb{A}=\mathbb{K}\left[q_{1}, \ldots, q_{n}\right]$ where $\mathbb{K}$ is a field or $\mathbb{A}=\mathbb{Z} /(2)$.

Definition 3. The exterior finite difference operator, denoted $\Delta$, acts on a weight function $w: \mathcal{H}_{k, n} \longrightarrow \mathbb{R}$ by producing a new weight function, denoted $\Delta w$, defined on the $(k+1)$-dimensional hypercubes in $\mathcal{H}_{k+1, n}$ by

$$
\Delta w=\left((\Delta w)_{i_{1}, \ldots, i_{k+1}}\right)_{1 \leq i_{1}<\cdots<i_{k+1} \leq n},
$$

where

$$
(\Delta w)_{i_{1}, \ldots, i_{k+1}}\left(\alpha_{1}, \ldots, \alpha_{n}\right)=\sum_{\nu=1}^{k+1}(-1)^{\nu-1} \Delta_{i_{\nu}} w_{i_{1}, \ldots, \hat{i_{\nu}}, \ldots, i_{k+1}}\left(\alpha_{1}, \ldots, \alpha_{n}\right) .
$$

Proposition 1. The exterior difference operator $\Delta$ satisfies $\Delta^{2}=0$.

Definition 4. Let $w$ be a weight function on hypercubes. We say that $w$ is closed if $\Delta w=0$ and exact if $\exists \rho$ such that $w=\Delta \rho$.

By linearity, we can extend $w$ and $\Delta w$ to hypercubic configurations.

Theorem 2. (Discrete version of Stokes' theorem) For every $k$-dimensional hypercubic configuration $\mathbf{P}$ in $\mathbb{R}^{n}$ and every weight function $w$ defined on $(k-1)$ dimensional hypercubic configurations in $\mathbb{R}^{n},(\Delta w)(\mathbf{P})=w(\partial \mathbf{P})$.

As in 123 , it is more useful to write $(\Delta w)(\mathbf{P})$ in the form $W(\mathbf{P})$, where $W$ is a given weight function and $\mathbf{P}$ is an arbitrary hypercubic configuration. Then, the right member $w(\partial \mathbf{P})$ can be described as an algorithm to compute $W(\mathbf{P})$ using another weight function $w$, applied to the boundary $\partial \mathbf{P}$.

By analogy with the finite difference calculus, we can use the suggestive notation $w=\Sigma W$. Then $(\Delta w)(\mathbf{P})=w(\partial \mathbf{P})$ can be rewritten as,

$$
W(\mathbf{P})=(\Sigma W)(\partial \mathbf{P}) .
$$

Before defining the exterior summation operator $\Sigma$ we need to introduce the partial summation operator $\Sigma_{i}$ : for $i=1, \ldots, n, \Sigma_{i}$ applied on a function $f$ produces a function $\Sigma_{i} f: \mathbb{Z}^{n} \longrightarrow \mathbb{R}$ defined by

$$
\Sigma_{i} f\left(\alpha_{1}, \ldots, \alpha_{n}\right)= \begin{cases}\sum_{\nu_{i}=0}^{\alpha_{i}-1} f\left(\alpha_{1}, \ldots, \nu_{i}, \ldots, \alpha_{n}\right) & \text { if } \alpha_{i}>0, \\ 0 & \text { if } \alpha_{i}=0, \\ -\sum_{\nu_{i}=\alpha_{i}}^{-1} f\left(\alpha_{1}, \ldots, \nu_{i}, \ldots, \alpha_{n}\right) & \text { if } \alpha_{i}<0 .\end{cases}
$$


Lemma 2. The operators $\Delta_{i}$ and $\Sigma_{i}$ are related as follows

$$
\Delta_{i} \Sigma_{i}=\mathrm{id} \quad \text { and } \quad \Sigma_{i} \Delta_{i}=\mathrm{id}-\mathrm{eval}_{i, 0},
$$

where eval $_{i, 0}$ is the evaluation operator of the $i$-th component at the origin, defined by eval $_{i, 0} f\left(\alpha_{1}, \ldots, \alpha_{i}, \ldots, \alpha_{n}\right)=f\left(\alpha_{1}, \ldots, 0, \ldots, \alpha_{n}\right)$. Moreover, if $i \neq j$, then $\Delta_{j} \Sigma_{i}=\Sigma_{i} \Delta_{j}$.

The vector space of weight functions on $k$-dimensional hypercubes in $\mathbb{R}^{n}$ is denoted by $\mathbb{W}_{k, n}=\left\{w \mid w: \mathcal{H}_{k, n} \longrightarrow \mathbb{R}\right\}$. We are now ready to define the exterior summation operator $\Sigma$.

Definition 5. The exterior finite summation operator $\Sigma: \mathbb{W}_{k, n} \longrightarrow \mathbb{W}_{k-1, n}$ is defined by

$$
\begin{aligned}
&(\Sigma W)_{j_{1}, \ldots, j_{k-1}}\left(\alpha_{1}, \ldots, \alpha_{n}\right) \\
&=(-1)^{k-1}\left(\sum_{j_{k-1}<i} \Sigma_{i} W_{j_{1}, \ldots, j_{k-1}, i}\left(\alpha_{1}, \ldots, \alpha_{i}, 0, \ldots, 0\right)\right) .
\end{aligned}
$$

In order to have (1), we made the assumption that $W$ was exact. In general, this condition is difficult to check. Fortunately, in our context of everywhere defined weight functions on hypercubes, exactness is equivalent to closedness, which is a much easier condition to check. This is precisely the content of the following discrete version of Poincaré lemma. It is noteworthy that our proof is non inductive in contrast with the corresponding proof given in [4] in the context of difference forms.

Theorem 3. Let $W$ be a weight function defined on hypercubes. Then $W$ is closed if and only if $W$ is exact.

Proof. Obviously, $W=\Delta w \Longrightarrow \Delta W=\Delta^{2} w=0$. To establish the converse, we assume that $\Delta W=0$ and show that $W$ is exact. It suffices to see that $\Delta \Sigma W+\Sigma \Delta W=W$, since, taking $w=\Sigma W$, we will find $\Delta w=\Delta \Sigma W=$ $\Delta \Sigma W+0=\Delta \Sigma W+\Sigma 0=\Delta \Sigma W+\Sigma \Delta W=W$.

On one hand, we have,

$$
\begin{aligned}
(\Delta \Sigma W)_{i_{1}, \ldots, i_{k}}\left(\alpha_{1}, \ldots, \alpha_{n}\right) & \\
= & \sum_{1 \leq \nu \leq k}(-1)^{\nu-1} \Delta_{i_{\nu}}(\Sigma W)_{i_{1}, \ldots, \hat{i_{\nu}}, \ldots, i_{k}}\left(\alpha_{1}, \ldots, \alpha_{n}\right) \\
= & \sum_{1 \leq \nu \leq k, i_{k}<i \leq n}(-1)^{\nu-1+k-1} \Delta_{i_{\nu}} \Sigma_{i} W_{i_{1}, \ldots, \hat{i_{\nu}}, \ldots, i_{k}, i}\left(\alpha_{1}, \ldots, \alpha_{i}, 0, \ldots, 0\right) \\
& \quad+\sum_{\nu=k, i=i_{k}}(-1)^{2 k-2} \Delta_{i_{k}} \Sigma_{i} W_{i_{1}, \ldots, i_{k-1}, \hat{i_{k}, i}}\left(\alpha_{1}, \ldots, \alpha_{i}, 0, \ldots, 0\right) \\
= & +\Delta_{i_{k}} \Sigma_{i_{k}} W_{i_{1}, \ldots, i_{k}}\left(\alpha_{1}, \ldots, \alpha_{i_{k}}, 0, \ldots, 0\right), \quad \text { say } \\
= & S+W_{i_{1}, \ldots, i_{k}}\left(\alpha_{1}, \ldots, \alpha_{i_{k}}, 0, \ldots, 0\right) .
\end{aligned}
$$


On the other hand,

$$
\begin{aligned}
& (\Sigma \Delta W)_{i_{1}, \ldots, i_{k}}\left(\alpha_{1}, \ldots, \alpha_{n}\right) \\
& =\sum_{i_{k}<i \leq n, 1 \leq \nu \leq k}(-1)^{k+\nu-1} \Sigma_{i} \Delta_{i_{\nu}} W_{i_{1}, \ldots, \hat{i_{\nu}}, \ldots, i_{k}, i}\left(\alpha_{1}, \ldots, \alpha_{i}, 0, \ldots, 0\right) \\
& \quad+\sum_{i_{k}<i \leq n}(-1)^{2 k} \Sigma_{i} \Delta_{i} W_{i_{1}, \ldots, i_{k}, \hat{i}}\left(\alpha_{1}, \ldots, \alpha_{i}, 0, \ldots, 0\right) \\
& \quad=-S+\sum_{i_{k}<i \leq n} \Sigma_{i} \Delta_{i} W_{i_{1}, \ldots, i_{k}}\left(\alpha_{1}, \ldots, \alpha_{i}, 0, \ldots, 0\right) .
\end{aligned}
$$

Hence, using Lemma 2,

$$
\begin{aligned}
& (\Delta \Sigma W)_{i_{1}, \ldots, i_{k}}\left(\alpha_{1}, \ldots, \alpha_{n}\right)+(\Sigma \Delta W)_{i_{1}, \ldots, i_{k}}\left(\alpha_{1}, \ldots, \alpha_{n}\right) \\
& =W_{i_{1}, \ldots, i_{k}}\left(\alpha_{1}, \ldots, \alpha_{i_{k}}, 0, \ldots, 0\right)+\sum_{i_{k}<i \leq n} \Sigma_{i} \Delta_{i} W_{i_{1}, \ldots, i_{k}}\left(\alpha_{1}, \ldots, \alpha_{i}, 0, \ldots, 0\right) \\
& =W_{i_{1}, \ldots, i_{k}}\left(\alpha_{1}, \ldots, \alpha_{i_{k}}, 0, \ldots, 0\right) \\
& \quad+\sum_{i_{k}<i \leq n}\left(W_{i_{1}, \ldots, i_{k}}\left(\alpha_{1}, \ldots, \alpha_{i}, 0, \ldots, 0\right)-W_{i_{1}, \ldots, i_{k}}\left(\alpha_{1}, \ldots, \alpha_{i-1}, 0, \ldots, 0\right)\right) \\
& =W_{i_{1}, \ldots, i_{k}}\left(\alpha_{1}, \ldots, \alpha_{n}\right) .
\end{aligned}
$$

We have the following useful variant of discrete Stokes' theorem.

Theorem 4. (Variant of the discrete Stokes' theorem) If $W$ is closed, then for every hypercubic configuration $\mathbf{P}$, we have $W(\mathbf{P})=(\Sigma W)(\partial \mathbf{P})$.

Note that the weight $\Sigma W$ is only one of an infinite number of possible weights for the right-hand side of the equality in Theorem 4

Corollary 1. Every weight function $\Lambda: \mathcal{H}_{k-1, n} \longrightarrow \mathbb{R}$ satisfying

$$
W(\mathbf{P})=\Lambda(\partial \mathbf{P})
$$

is of the form $\Lambda=\Sigma W+\Theta$, where $\Theta$ is an exact weight function, that is of the form $\Theta=\Delta \Omega$, where $\Omega: \mathcal{H}_{k-2, n} \longrightarrow \mathbb{R}$.

\section{Examples and Applications}

Theorem 4 gives rise to many applications for the reduction of the computation of a weight function on hypercubic configurations to the computation of another weight function on the boundary of the configurations.

Our discrete version of Stokes' theorem generalizes the classical one in the particular case of hypercubic configurations. Indeed, let $\omega$ be a $(k-1)$ - form in $\mathbb{R}^{n}$ and define the weight function $w: \mathcal{H}_{k-1, n} \longrightarrow \mathbb{R}$ by $w(H)=\int_{H} \omega$ for any 
$(k-1)$-dimensional hypercube $H$. Then, it can be checked that $\Delta w: \mathcal{H}_{k, n} \longrightarrow \mathbb{R}$ satisfies $(\Delta w)(K)=\int_{K} d \omega$. Hence, $(\Delta w)(K)=w(\partial K)$ is equivalent to the classical Stokes' theorem for hypercubes. But when a weight function is not written in the form $w(H)=\int_{H} \omega$ or have values in an arbitrary commutative ring, Theorems 2 and 4 can be directly applied using no integration.

\subsection{The Special Case $k=n$}

Consider an arbitrary weight function $W \in \mathbb{W}_{n, n}$. Such a weight function is always closed since it reduces to a single function $W_{1, \ldots, n}$ defined on $n$-dimensional hypercubes in $\mathbb{R}^{n}$ and $\Delta W$ is an empty sum. Writing $\Phi=W_{1, \ldots, n}$, then without further hypotheses on $\Phi$, Theorem 4 reduces to the following:

Proposition 2. Let $\Phi: \mathcal{H}_{n, n} \longrightarrow \mathbb{R}$. Then for any $n$-dimensional hypercubic configuration $\mathbf{P}$ in $\mathbb{R}^{n}$, we have $\Phi(\mathbf{P})=(\Sigma \Phi)(\Delta \mathbf{P})$. Explicitly,

$$
\begin{aligned}
& (\Sigma \Phi)_{j_{1}, \ldots, j_{n-1}}\left(\alpha_{1}, \ldots, \alpha_{n}\right) \\
& = \begin{cases}(-1)^{n-1} \Sigma_{n} \Phi\left(\alpha_{1}, \ldots, \alpha_{n}\right) & \text { if }\left(j_{1}, j_{2}, \ldots, j_{n-1}\right)=(1,2, \ldots, n-1), \\
0 & \text { otherwise }\end{cases}
\end{aligned}
$$

Take $n=3$ and consider, for example, the computation of the moment of inertia, $\mathrm{I}(\mathbf{P})$, relative to the $x_{1}$-axis, of a 3 -dimensional cubical configuration $\mathbf{P}=\sum_{k=1}^{N} H_{k}$, which is a union of $N$ distinct unit cubes each having mass 1 . Taking $\Phi\left(\alpha_{1}, \alpha_{2}, \alpha_{3}\right)=\mathrm{I}\left(\operatorname{Pix}_{123}\left(\alpha_{1}, \alpha_{2}, \alpha_{3}\right)\right)=\left(\alpha_{2}+1 / 2\right)^{2}+\left(\alpha_{3}+1 / 2\right)^{2}+1 / 6$, we find, using Proposition 2 , that $\Sigma \Phi: \mathcal{H}_{2,3} \rightarrow \mathbb{R}$ is given by

$$
\begin{aligned}
& (\Sigma \Phi)_{12}\left(\alpha_{1}, \alpha_{2}, \alpha_{3}\right)=\Sigma_{3} \Phi\left(\alpha_{1}, \alpha_{2}, \alpha_{3}\right)=\alpha_{3}\left(\alpha_{2}^{2}+\alpha_{2}+1 / 3 \alpha_{3}^{2}+1 / 3\right), \\
& (\Sigma \Phi)_{13}\left(\alpha_{1}, \alpha_{2}, \alpha_{3}\right)=0, \quad(\Sigma \Phi)_{23}\left(\alpha_{1}, \alpha_{2}, \alpha_{3}\right)=0 .
\end{aligned}
$$

Hence $\mathrm{I}(\mathbf{P})=(\Sigma \Phi)(\partial \mathbf{P})$. This is often much shorter to compute since the number of squares in $\partial \mathbf{P}$ is generally $O\left(N^{2 / 3}\right)$ compared to the number $N$ of cubes in P.

The moment of inertia of $\mathbf{P}$ relative to any axis (oblique or not) can be computed in a similar way. For the center of gravity $g(\mathbf{P})=\left(\bar{x}_{1}, \bar{x}_{2}, \bar{x}_{3}\right)$ of the cubical configuration $\mathbf{P}$, one can take the vector-valued function $\Phi: \mathcal{H}_{3,3} \rightarrow \mathbb{R}^{3}$, defined by

$$
\Phi\left(\alpha_{1}, \alpha_{2}, \alpha_{3}\right)=g\left(\operatorname{Pix}_{123}\left(\alpha_{1}, \alpha_{2}, \alpha_{3}\right)\right)=\left(\alpha_{1}+1 / 2, \alpha_{2}+1 / 2, \alpha_{3}+1 / 2\right) .
$$

In this case, $\Sigma \Phi: \mathcal{H}_{2,3} \rightarrow \mathbb{R}^{3}$ is given by

$$
\begin{aligned}
& (\Sigma \Phi)_{12}\left(\alpha_{1}, \alpha_{2}, \alpha_{3}\right)=\left(\left(\alpha_{1}+1 / 2\right) \alpha_{3},\left(\alpha_{2}+1 / 2\right) \alpha_{3}, 1 / 2 \alpha_{3}^{2}\right), \\
& (\Sigma \Phi)_{13}\left(\alpha_{1}, \alpha_{2}, \alpha_{3}\right)=\mathbf{0}, \quad(\Sigma \Phi)_{23}\left(\alpha_{1}, \alpha_{2}, \alpha_{3}\right)=\mathbf{0} .
\end{aligned}
$$

and we have $g(\mathbf{P})=1 / N(\Sigma \Phi)(\partial \mathbf{P})$.

For the special case $k=n=2$, taking $\Phi(\mathbf{P})=\iint_{\mathbf{P}} f(x, y) d x d y$ and using the graphical convention of Figure 1, Proposition 2 generalizes the vertical algorithm 
( $V$-algo) given in [123]: for a lattice polyomino $\mathbf{P} \subseteq \mathbb{R}^{2}$ given by its contour $\left(x_{0}, y_{0}\right),\left(x_{1}, y_{1}\right), \ldots,\left(x_{n}, y_{n}\right)=\left(x_{0}, y_{0}\right)$, then

$\iint_{\mathbf{P}} f(x, y) d x d y=\sum_{\rightarrow} \Phi_{r}\left(x_{i}, y_{i}\right)+\sum_{\uparrow} \Phi_{u}\left(x_{i}, y_{i}\right)+\sum_{\leftarrow} \Phi_{l}\left(x_{i}, y_{i}\right)+\sum_{\downarrow} \Phi_{d}\left(x_{i}, y_{i}\right)$,

where functions $\Phi_{r}, \Phi_{u}, \Phi_{l}, \Phi_{d}$ are given by :

$$
\Phi_{r}=0, \quad \Phi_{d}=\int_{0}^{1} f_{1}(x, y+t) d t, \quad \Phi_{l}=0, \quad \Phi_{u}=-\int_{0}^{1} f_{1}(x, y-t) d t,
$$

where $f_{1}(x, y)=\int^{x} f(u, y) d u$. By Corollary 1, the general form for $\Lambda$ such that $\iint_{\mathbf{P}} f(x, y) d x d y=\Lambda(\partial H)$, is given by $\Lambda=\Sigma \Phi+\Theta$, where $\Sigma \Phi$ is given (in the context of [1 2 3 ] ) by the right-hand side of the above equation and $\Theta=\Delta \Omega$, with an arbitrary $\Omega: \mathcal{H}_{0,2} \longrightarrow \mathbb{R}$, that is, $\Omega: \mathbb{Z} \times \mathbb{Z} \longrightarrow \mathbb{R}$. Therefore, writing $\Omega=\Omega\left(\alpha_{1}, \alpha_{2}\right)$, then $\Theta: \mathcal{H}_{1,2} \longrightarrow \mathbb{R}$, where

$$
\begin{aligned}
& \Theta_{1}=(\Delta \Omega)_{1}=(-1)^{1-1} \Delta_{1} \Omega\left(\alpha_{1}, \alpha_{2}\right)=\Omega\left(\alpha_{1}+1, \alpha_{2}\right)-\Omega\left(\alpha_{1}, \alpha_{2}\right), \\
& \Theta_{2}=(\Delta \Omega)_{2}=(-1)^{1-1} \Delta_{2} \Omega\left(\alpha_{1}, \alpha_{2}\right)=\Omega\left(\alpha_{1}, \alpha_{2}+1\right)-\Omega\left(\alpha_{1}, \alpha_{2}\right) .
\end{aligned}
$$

This corresponds to Corollary 8 of 2 using the convention of Figure 1] For an example involving weight values in the ring $\mathbb{Z}\left[q_{1}, \ldots, q_{n}\right]$, consider the weight function $\Phi=W_{1, \ldots, n}$, where $\Phi\left(\alpha_{1}, \ldots, \alpha_{n}\right)=q_{1}^{\alpha_{1}} \ldots q_{n}^{\alpha_{n}}$. Then,

$$
(\Sigma \Phi)_{1, \ldots, n-1}\left(\alpha_{1}, \ldots, \alpha_{n}\right)=(-1)^{n-1} q_{1}^{\alpha_{1}} \ldots q_{n-1}^{\alpha_{n-1}}\left[\alpha_{n}\right]_{q_{n}},
$$

where $[\alpha]_{q}=1+q+\cdots+q^{\alpha-1}$ is the $q$-analogue of $\alpha$. This generalizes the concept of $q$-area introduced in $1 \frac{1}{12} 3$.

\subsection{The Case $k<n$}

We focus now on some specific families of weights in the context of Theorem 4 for $k<n$. Consider weights of the form

$$
W_{i_{1}, \ldots, i_{k}}\left(\alpha_{1}, \ldots, \alpha_{n}\right)=c_{i_{1}, \ldots, i_{k}},
$$

depending only on the directions $i_{1}, \ldots, i_{k}$ of the hypercubes. The condition $\Delta W=0$ is satisfied and after some computations

$$
(\Sigma W)_{j_{1}, \ldots, j_{k-1}}\left(\alpha_{1}, \ldots, \alpha_{n}\right)=(-1)^{k-1} \sum_{j_{k-1}<i} c_{j_{1}, \ldots, j_{k-1}, i} \alpha_{i} .
$$

For example, if $c_{i_{1}, \ldots, i_{k}}=1$ for all $i_{1}, \ldots, i_{k}$ (= volume of an hypercube), then $W(\mathbf{P})$ is the signed volume of the hypercubic configuration $\mathbf{P}$. In other words, if $\mathbf{P}=\sum_{i=1}^{m} n_{i} H_{i}$ then $W(\mathbf{P})=\sum_{i=1}^{m} n_{i}$. We then obtain,

$$
(\Sigma W)_{j_{1}, \ldots, j_{k-1}}\left(\alpha_{1}, \ldots, \alpha_{n}\right)=(-1)^{k-1}\left(\alpha_{j_{k-1}+1}+\cdots+\alpha_{n}\right) .
$$


This generalizes the $H$-algo for the area of a polyomino given in [1 2 3] .

Consider now weights of the form

$$
W_{i_{1}, \ldots, i_{k}}\left(\alpha_{1}, \ldots, \alpha_{n}\right)=f\left(\alpha_{1}, \ldots, \alpha_{n}\right)
$$

depending only on the principal corner of the hypercubes. We have two cases to consider:

(i) If $0 \leq k \leq n-2$, then it can be shown that $\Delta W=0$ if and only if

$$
f\left(\alpha_{1}, \ldots, \alpha_{n}\right)= \begin{cases}\varphi\left(\alpha_{1}+\cdots+\alpha_{n}\right) & \text { if } k \text { odd } \\ \text { a constant } c & \text { if } k \text { even }\end{cases}
$$

where $\varphi: \mathbb{Z} \rightarrow \mathbb{R}$ is an arbitrary function. It turns out that

$$
\begin{aligned}
& (\Sigma W)_{j_{1}, \ldots, j_{k-1}}\left(\alpha_{1}, \ldots, \alpha_{n}\right) \\
& = \begin{cases}F\left(\alpha_{1}+\cdots+\alpha_{n}\right)-F\left(\alpha_{1}+\cdots+\alpha_{j_{k-1}}\right) & \text { if } k \text { odd } \leq n-2, \\
c \cdot\left(\alpha_{j_{k-1}+1}+\cdots+\alpha_{n}\right) & \text { if } k \text { even } \leq n-2,\end{cases}
\end{aligned}
$$

where $F(x)=\sum_{x} \varphi(x), 1 \leq j_{1}<\cdots<j_{k-1} \leq n$.

(ii) If $k=n-1$ and $f\left(\alpha_{1}, \ldots, \alpha_{n}\right)$ is a polynomial, then it can be shown that $\Delta W=0$ if and only if

$$
f\left(\alpha_{1}, \ldots, \alpha_{n}\right)=\left(I+\Delta_{2}-\Delta_{3}+\cdots+(-1)^{n-1} \Delta_{n}\right)^{\alpha_{1}} \Psi\left(\alpha_{2}, \ldots, \alpha_{n}\right),
$$

where $\Psi: \mathbb{Z}^{n-1} \rightarrow \mathbb{R}$ is an arbitrary polynomial.

\section{Concluding Remarks}

The results of this paper can be used to study many other statistics about hypercubic configurations in relation to parameters associated to their boundary. This is particularly true when the weight functions are polynomials written in the form

$$
W_{i_{1}, \ldots, i_{k}}\left(\alpha_{1}, \ldots, \alpha_{n}\right)=\sum_{p_{1}, \ldots, p_{n} \geq 0} c_{p_{1}, \ldots, p_{n}}^{i_{1}, \ldots, i_{k}}\left(\begin{array}{l}
\alpha_{1} \\
p_{1}
\end{array}\right) \ldots\left(\begin{array}{l}
\alpha_{n} \\
p_{n}
\end{array}\right)
$$

where $\left(\begin{array}{l}\alpha \\ p\end{array}\right)=\alpha(\alpha-1) \ldots(\alpha-p+1) / p$ !. Since $\Delta_{i}\left(\begin{array}{c}\alpha_{i} \\ p_{i}\end{array}\right)=\left(\begin{array}{c}\alpha_{i} \\ p_{i}-1\end{array}\right)$, we have

$$
\Delta W=0 \Longleftrightarrow \sum_{\nu=1}^{k+1}(-1)^{\nu-1} c_{p_{1}, \ldots, p_{j_{\nu}+1}, \ldots, p_{n}}^{j_{1}, \ldots, \hat{j}_{\nu}, \ldots, j_{k+1}}=0
$$

for every $j_{1}<\cdots<j_{k+1}$. Moreover, since $\sum_{i}\left(\begin{array}{c}\alpha_{i} \\ p_{i}\end{array}\right)=\left(\begin{array}{c}\alpha_{i} \\ p_{i}+1\end{array}\right)$, we have

$$
(\Sigma W)_{j_{1}, \ldots, j_{k-1}}\left(\alpha_{1}, \ldots, \alpha_{n}\right)=\sum_{p_{1}, \ldots, p_{n} \geq 0} \gamma_{p_{1}, \ldots, p_{n}}^{j_{1}, \ldots, j_{k-1}}\left(\begin{array}{l}
\alpha_{1} \\
p_{1}
\end{array}\right) \cdots\left(\begin{array}{l}
\alpha_{n} \\
p_{n}
\end{array}\right)
$$


where $\gamma_{0, \ldots, 0}^{j_{1}, \ldots, j_{k-1}}=0$ and for $\left(p_{1}, \ldots, p_{n}\right) \neq(0, \ldots, 0)$,

$$
\gamma_{p_{1}, \ldots, p_{n}}^{\left(j_{1}, \ldots, j_{k-1}\right)}=(-1)^{k-1} \sum_{j_{k-1}<i} c_{p_{1}, \ldots, p_{\nu_{0}}-1,0, \ldots, 0}^{\left(j_{1}, \ldots, j_{k-1}, i\right)}
$$

where $\nu_{0}=\min \left\{\nu \mid p_{\nu} \neq 0\right\}$.

Acknowledgements. The authors wish to thank the anonymous referees for the careful reading of the paper and their valuable comments.

\section{References}

1. Brlek, S., Labelle, G., Lacasse, A.: Incremental algorithms based on discrete Green theorem. In: Nyström, I., Sanniti di Baja, G., Svensson, S. (eds.) DGCI 2003. LNCS, vol. 2886, pp. 277-287. Springer, Heidelberg (2003)

2. Brlek, S., Labelle, G., Lacasse, A.: The discrete Green theorem and some applications in discrete geometry. Theoret. Comput. Sci. 346(2), 200-225 (2005)

3. Lacasse, A.: Contributions à l'analyse de figures discrètes en dimension quelconque. PhD thesis, Université du Québec à Montréal (September 2008)

4. Mansfield, E.L., Hydon, P.E.: Difference forms. Found. Comp. Math. (2007), http://personal.maths.surrey.ac.uk/st/P.Hydon/

5. Desbrun, M., Kanso, E., Tong, Y.: Discrete differential forms for computational modeling. In: Discrete differential geometry. Oberwolfach Semin, vol. 38, pp. 287-324. Birkhäuser, Basel (2008)

6. Kaczynski, T., Mischaikow, K., Mrozek, M.: Computing homology. Homology Homotopy Appl 5(2), 233-256 (2003); (electronic) Algebraic topological methods in computer science (Stanford, CA, 2001)

7. González-Díaz, R., Real, P.: On the cohomology of 3D digital images. Discrete Appl. Math. 147(2-3), 245-263 (2005)

8. Spivak, M.: Calculus on manifolds. A modern approach to classical theorems of advanced calculus. W. A. Benjamin, Inc., New York (1965) 